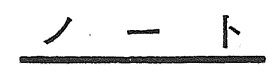

\title{
Structures and Hypotensive Effect of Flavonoid Glycosides in Unshiu Peeling. $\mathrm{II}^{\dagger}$.
}

\author{
Hiroyasu Kumamoto, Yoshiharu Matsubara, Yoshitomi IIzuKA*, \\ Kozo OKAMOTO** and Katsumi YoKoI*** \\ Department of Applied Chemistry, Faculty of Science and Engineering, Kinki University, \\ * Research Institute of Food Science, Kinki University, ** Kinki University School of \\ Medicine and ***Japan Sangaria Beverage Co. Ltd.
}

\begin{abstract}
We describe the structures and hypotensive effects of three additional glycosides $(1,2$ and 3 ) obtained through further investigation on unshiu peelings. The structures 3 -hydroxy- $3^{\prime}, 4^{\prime}, 5,6,7$, 8-hexamethoxyflavone 3- $\beta$-D-glucoside (1), limocitrin 3-O-[5- $\alpha$-glucosyl-3-hydroxy-3-methylglutaryl $(1 \rightarrow 2)]-\beta$-glucoside (2) and narirutin- $4^{\prime}$-glucoside (3) were established by UV, ${ }^{1} \mathrm{H}-\mathrm{NMR}$ and ${ }^{13} \mathrm{C}-\mathrm{NMR}$ spectroscopy, and chemical evidence. Compounds 1 and 3 showed strong depressive effects, when intravenously injected with stroke-prone spontaneously hypotensive rats $(0.5 \sim 1.0 \mathrm{mg}$ dose $/ 100 \mathrm{~g}$ body weight). Among these compounds, 1 was a new flavonoid glycoside, and 2 and 3 were found for the first time in unshiu.
\end{abstract}

In the previous paper, we reported six flac vonoid glycosides, limocitrin $3-\alpha-\mathrm{L}-$ rhamnoside, apigenin 3,6-di-C-glucoside, narirutin, rutin and narcissin isolated from Citrus unshiu peelings and their depressive effect on blood pressure ${ }^{1)}$. We wish to describe herein the structures and hypotensive effects of three additional glycosides (1, 2 and 3) which have been obtained by further investigation on unshiu peelings.

Thus, these glycosides were isolated by re= peated silica gel column chromatography of the crude flavonoid fractions which had been pres pared $^{11}$ by gel filtration. The molecular weight of 1 was found to be 580 by FAB-MS which showed characteristic peaks at $m / z 419$ [aglys cone $+\mathrm{H}]^{+}, m / z 581[\mathrm{M}+\mathrm{H}]^{+}$and $m / z 603[\mathrm{M}$ $+\mathrm{Na}]^{+}$. Maximum absorptions at 254 and 343 $\mathrm{nm}$ were shown in its UV spectrum in ethanol. These absorptions did not shift significantly upon addition of sodium acetate or aluminum chloride. These facts suggest that 1 did not have functional groups such as 7-hydroxy-, $4^{\prime}$ hydroxy-4-oxo-, 3-hydroxy-4-oxo- and o-di= hydroxyl-systems ${ }^{3}$. The ${ }^{1} \mathrm{H}-\mathrm{NMR}$ spectrum of 1 indicates the presence of aromatic signals of an

$\dagger$ Studies on Physiologically Active Substances in Citrus Peel. Part VII. For Part VI, see ref. 2).
ABX type at $\delta 7.87(J=2$ $\mathrm{Hz}), 6.96(J=9 \mathrm{~Hz})$ and $7.85(J=2$ and $9 \mathrm{~Hz})$, re= spectively, assignable to $\mathrm{H}_{2}{ }^{\prime}$, $\mathrm{H}_{5}{ }^{\prime}$ and $\mathrm{H}_{6}{ }^{\prime}$, six methoxy groups at $\delta 3.89 \sim 4.08$, and an anomeric proton of a $\beta$-glucoside moiety at $\delta 4.88$ $(J=7 \mathrm{~Hz})$. The structure

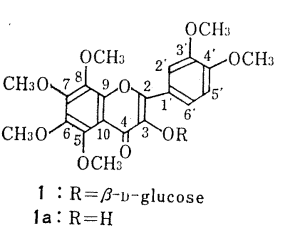

Fig.-1 Structure of 1 and $1 \mathrm{a}$. of a hexamethoxyflavonol glycoside is supported by the appearance of twenty-seven signals including six carbon signals of $\beta$-gluco $=$ side and six methoxy carbon signals in the ${ }^{13} \mathrm{C}-\mathrm{NMR}$ spectrum. Acid hydrolysis of 1 gave D-glucose $\left([\alpha]_{\mathrm{D}}+42.2^{\circ}, \quad c=0.1, \mathrm{H}_{2} \mathrm{O}\right)$ and aglycone (1 a). The ${ }^{1} \mathrm{H}-\mathrm{NMR}$ spectrum of $1 \mathbf{a}$ was similar to that of 1 except for the absence of signals due to the sugar protons of 1 . The UV spectrum of $1 \mathrm{a}$ in ethanol shows maximum absorptions at 258 and $369 \mathrm{~nm}$, which exhibit no shift on the addition of sodium acetate. However, the band I $(369 \mathrm{~nm})$ indicates a bathochromic shift of $67 \mathrm{~nm}$ when aluminum chloride was added, suggesting the presence of 3-hydroxy-4-oxo- system $^{3)}$ in 1 a. Therefore, the structure of 3 -hydroxy-3', $4^{\prime}, 5,6,7$, 8-hexamethoxyflavone ${ }^{4)}$ was assigned to $1 \mathbf{a}$ and 1 was determined as 3 -hydroxy- $3^{\prime}, 4^{\prime}, 5,6,7$, 
Table-I Hypotensive effect.

\begin{tabular}{|c|c|}
\hline Compound & $\begin{array}{l}\text { Maximal decrease of blood } \\
\text { pressure (mmHg) } \\
\text { [Time after administration (h) }\end{array}$ \\
\hline 1 & $-24^{\mathrm{a}} \quad[0.5]$ \\
\hline 2 & $-47^{\mathrm{b}} \quad[1]$ \\
\hline
\end{tabular}

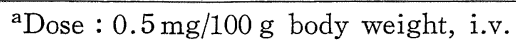

${ }^{\mathrm{b}}$ Dose $: 1.0 \mathrm{mg} / 100 \mathrm{~g}$ body weight, i.v.

8-hexamethoxyflavone $3-\beta-D$-glucoside.

Compound 2 was identified to be limocitrin 3-O-\{[5- $\alpha$-glucosyl-3-hydroxy-3-methylglutaryl $(1 \rightarrow 2)]-\beta$-glucoside\} by comparing its UV, FAB-MS and ${ }^{1} \mathrm{H}-\mathrm{NMR}$ spectra with those of an authentic sample previously isolated from lemon peels: ${ }^{5}$.

The UV spectral data, specific rotation and melting point of 3 were identical with those of an authentic specimen of narirutin-4'-glucos side $^{6}$.

Depressive effect on blood pressure were examined for these flavonoid glycosides 1 and 3 , and the results are shown as average values of triplicates (Table-1). The blood pressure of stroke-prone spontaneously hypotensive rats (SHR-SP) showed the maximum decrease of 24 $\mathrm{mmHg}$ for 1 ( $0.5 \mathrm{mg}$ dose $/ 100 \mathrm{~g}$ body weight) at $30 \mathrm{~min}$ and $47 \mathrm{mmHg}$ for 3 (1.0 $\mathrm{mg}$ dose/ $100 \mathrm{~g}$ body weight) at $1 \mathrm{~h}$ after the intravenous administration. Accordingly, flavonoid glycos sides 1 and 3 from unshiu peelings are regard= ed as important constituents having depressive effect for SHR-SP.

Among these compounds isolated by the present study, 1 was found to be a new flac vonoid glycoside, and also 2 and 3 were found for the first time in unshiu.

\section{Experimental}

Analytical instruments. The optical rotation was measured with a Japan Spectroscopic DIP140. FAB-MS spectra were obtained with JEOL JMS-HX 100 and JMA-DA 5000 instru= ments under xenon bombardment $(6.0 \mathrm{keV})$. UV spectra were taken with a Hitachi 323 visible-ultraviolet autoanalyzer. NMR spectra were measured with a JEOL-FX 200 in $\mathrm{CDCl}_{3}$, $\mathrm{CD}_{3} \mathrm{OD}$ or $\mathrm{DMSO}-d_{6}\left(200 \mathrm{MHz}\right.$ for ${ }^{1} \mathrm{H}$ and 50 $\mathrm{MHz}$ for ${ }^{13} \mathrm{C}$ ).

Separation and purification. Croude flas vonoids were obtained from unshiu peelings $(3100 \mathrm{~g})$ by the conventional method ${ }^{11}$. Cons stituents 1,2 and 3 were isolated by repeated chromatography of the gel-filtered fractions No. 8 , No. 13 , and No. $9^{1)}$, respectively in a column of silica gel with $\mathrm{CHCl}_{3}-\mathrm{MeOH}(9: 1)$ or $\mathrm{CHCl}_{3}-\mathrm{MeOH}-\mathrm{AcOH}-\mathrm{H}_{2} \mathrm{O} \quad(70: 15: 12: 3)$ as an eluant.

Acid hydrolysis of 1 and 3. A solution of $10 \mathrm{mg}$ of a sample in $5 \mathrm{ml}$ of $0.5 \mathrm{M}-\mathrm{HCl}$ was heated on a steam bath for $1 \mathrm{~h}$. Cooling, dilution with water, and extraction with EtOAc gave an aglycone.

Measurement of the blood depressive effect. Each substance was intravenously injected [0.5 $\sim 1.0 \mathrm{mg}$ dose $/ 100 \mathrm{~g}$ body weight $(220 \sim 250 \mathrm{~g})]$ in SHR-SP, whose blood pressure was mea sured chronologically using the tail pulse pickup method without anesthesia.

3-Hydroxy-3', 4', 5, 6, 7, 8-hexamethoxyflas vone 3- $\beta$-D-glucoside (1). Colorless needles (mp 111 $112^{\circ} \mathrm{C}$ ); FAB-MS : $m / z \quad 581 \quad[\mathrm{M}+$ $\mathrm{H}]^{+}, 603[\mathrm{M}+\mathrm{Na}]^{+}, 419[\text { aglycone }+\mathrm{H}]^{+}(100$ $\%)$; UV $\lambda_{\max }^{\mathrm{EtOH}} \mathrm{nm}: 254,269 \mathrm{~s}, 343 ; \lambda_{\max }^{\mathrm{AcONa}}$ $\mathrm{nm}: 256,270 \mathrm{~s}, 345 ; \lambda_{\max }^{\mathrm{AlCl}_{3}-\mathrm{EtOH}} \mathrm{nm}: 256,270 \mathrm{~s}$, $343 ;{ }^{1} \mathrm{H}-\mathrm{NMR} \delta_{\mathrm{Me}_{4} \mathrm{Si}}^{\mathrm{CDCl}_{3}}: 3.89 \mathrm{ppm}(3 \mathrm{H}, \mathrm{s}, \mathrm{OMe})$, $3.90(3 \mathrm{H}, \mathrm{s}, \mathrm{OMe}), 3.92(6 \mathrm{H}, \mathrm{s}, \mathrm{OMe} \times 2)$, $3.97(3 \mathrm{H}, \mathrm{s}, \mathrm{OMe}), 4.08$ ( $3 \mathrm{H}, \mathrm{s}, \mathrm{OMe}), 4.88$ $\left[1 \mathrm{H}, \mathrm{d}, J=7 \mathrm{~Hz}, \beta-\mathrm{Glc}\left(\mathrm{H}_{1^{\prime \prime}}\right)\right], 6.96(1 \mathrm{H}, \mathrm{d}$, $\left.J=9 \mathrm{~Hz}, \mathrm{H}_{5^{\prime}}\right), 7.85\left(1 \mathrm{H}\right.$, d.d, $J=2,9 \mathrm{~Hz}, \mathrm{H}_{6^{\prime}}{ }^{\prime}$, $7.87\left(1 \mathrm{H}, \mathrm{d}, J=2 \mathrm{~Hz}, \mathrm{H}_{2^{\prime}}\right) ;{ }^{13} \mathrm{C}-\mathrm{NMR} \delta_{\mathrm{Me}_{4} \mathrm{Si}}^{\mathrm{CD}_{3}}$ : $55.6 \mathrm{ppm}(\mathrm{q}, \mathrm{OMe}), 60.7\left(\mathrm{t}, \mathrm{C}_{6^{\prime \prime}}\right), 61.3(\mathrm{q}$; $\mathrm{OMe}), 61.4(\mathrm{q}, \mathrm{OMe}), 61.7(\mathrm{q}, \mathrm{OMe} \times 2), 61.8$ $(\mathrm{q}, \mathrm{OMe}), 69.8\left(\mathrm{~d}, \mathrm{C}_{4^{\prime \prime}}\right), 74.3\left(\mathrm{~d}, \mathrm{C}_{2^{\prime \prime}}\right), 76.5$ $\left(\mathrm{d}, \mathrm{C}_{3^{\prime \prime}}{ }^{\prime \prime}\right), 77.4\left(\mathrm{~d}, \mathrm{C}_{5^{\prime \prime}}\right), 100.6\left(\mathrm{~d}, \mathrm{C}_{1^{\prime \prime}}\right), 100.6$ $\left(\mathrm{s}, \mathrm{C}_{10}\right), 111.4\left(\mathrm{~d}, \mathrm{C}_{2^{\prime}}\right), 112.5\left(\mathrm{~d}, \mathrm{C}_{5^{\prime}}\right), 121.4$ $\left(\mathrm{d}, \mathrm{C}_{6^{\prime}}\right), 122.8\left(\mathrm{~s}, \mathrm{C}_{\mathrm{1}^{\prime}}\right), 135.5\left(\mathrm{~s}, \mathrm{C}_{8}\right), 137.4$ $\left(\mathrm{s}, \mathrm{C}_{3}\right), 143.3\left(\mathrm{~s}, \mathrm{C}_{9}\right), 146.1\left(\mathrm{~s}, \mathrm{C}_{6}\right), 147.2(\mathrm{~s}$, $\left.\mathrm{C}_{7}\right), 148.0\left(\mathrm{~s}, \mathrm{C}_{5}\right), 150.7\left(\mathrm{~s}, \mathrm{C}_{2}\right), 150.8(\mathrm{~s}$, $\left.\mathrm{C}_{3^{\prime}}\right), 153.3\left(\mathrm{~s}, \mathrm{C}_{4^{\prime}}\right), 172.1\left(\mathrm{~s}, \mathrm{C}_{4}\right)$.

3-Hydroxy-3', 4', 5, 6, 7, 8-hexamethoxyo flavone (1 a). Colorless needles (mp 138 140 ${ }^{\circ} \mathrm{C}$; lit. ${ }^{4)} 141 \sim 143^{\circ} \mathrm{C}$; UV $\lambda_{\max }^{\mathrm{EtOH}} \mathrm{nm}: 258,369$; $\lambda_{\max }^{\mathrm{AcONa}-\mathrm{EtOH}} \mathrm{nm}: 258,369 ; \lambda_{\max }^{\mathrm{AlCl}_{3}-\mathrm{EtOH}} \mathrm{nm}, 270$, $434 ;{ }^{1} \mathrm{H}-\mathrm{NMR} \delta_{\mathrm{Me}_{4} \mathrm{Si}_{3}}^{\mathrm{CDCl}_{3}}: 3.96 \mathrm{ppm}(3 \mathrm{H}, \mathrm{s}, \mathrm{OMe}$, $3.97(3 \mathrm{H}, \mathrm{s}, \mathrm{OMe}), 3.98(6 \mathrm{H}, \mathrm{s}, \mathrm{OMe} \times 2)$, $4.03(3 \mathrm{H}, \mathrm{s}, \mathrm{OMe}), 4.11(3 \mathrm{H}, \mathrm{s}, \mathrm{OMe}), 7.02$ $\left(1 \mathrm{H}, \mathrm{d}, J=8 \mathrm{~Hz}, \mathrm{H}_{5^{\prime}}\right), 7.90(1 \mathrm{H}, \mathrm{d}, J=2 \mathrm{~Hz}$, $\left.\mathrm{H}_{2^{\prime}}\right), 7.92\left(1 \mathrm{H}\right.$, d.d, $\left.J=2,8 \mathrm{~Hz}, \mathrm{H}_{6^{\prime}}\right)$. 
Narirutin-4'-glucoside (3). Pale yellow nee= dles (mp 196 $199^{\circ} \mathrm{C}$; lit. $^{6)} 189 \sim 200^{\circ} \mathrm{C}$ ); FABMS : $m / z \quad 765[\mathrm{M}+\mathrm{Na}]^{+}, 787\left[\mathrm{M}-\mathrm{H}+2 \mathrm{Na}^{+}\right.$; $\mathrm{UV} \lambda_{\max }^{\mathrm{EtOH}} \mathrm{nm}: 285,323 ; \lambda_{\max }^{\mathrm{AcONa}} \mathrm{EtOH}_{\mathrm{nm}}: 285$, 325; $\lambda_{\max }^{\mathrm{AlCl}_{3}-\mathrm{EtOH}} \mathrm{nm}: 309,342,386 ;{ }^{1} \mathrm{H}-\mathrm{NMR}$ ${ }_{{ }_{\mathrm{Me}} \mathrm{Si}}^{\mathrm{DMSO}-d_{6}}-: 1.10 \mathrm{ppm}(3 \mathrm{H}, \mathrm{d}, J=6 \mathrm{~Hz}$, rham $=$ nose-Me), $2.86\left(1 \mathrm{H}\right.$, d.d, $\left.J=3,17 \mathrm{~Hz}, \mathrm{H}_{3}\right)$, $4.54\left[1 \mathrm{H}, \mathrm{d}, J=1 \mathrm{~Hz}, \alpha\right.$-rhamnose $\left.\left(\mathrm{H}_{1^{\prime \prime}}\right)\right], 5.58$ $\left(1 \mathrm{H}\right.$, d.d, $\left.J=3,12 \mathrm{~Hz}, \mathrm{H}_{2}\right), 6.14(2 \mathrm{H}$, each s, $\left.\mathrm{H}_{6}, \mathrm{H}_{8}\right), 7.08\left(2 \mathrm{H}, \mathrm{d}, J=8 \mathrm{~Hz}, \mathrm{H}_{3^{\prime}, 5^{\prime}}\right), 7.46$ $\left(2 \mathrm{H}, \mathrm{d}, J=8 \mathrm{~Hz}, \mathrm{H}_{2^{\prime}, 6^{\prime}}\right), 11.70\left(1 \mathrm{H}, \mathrm{s}, \mathrm{C}_{5}-\right.$ $\mathrm{OH}) ;[\alpha]_{\mathrm{D}}:-61.1^{\circ}(c=0.46$, in Pyridine).

(Received Nov. 6, 1985)

\section{References}

1) Y. Matsubara, H. Kumamoto, Y. Iizuka, T. Murakami, K. Okamoto, H. Miyake, and K. Yokoi, Agric. Biol. Chem., 49, 909 (1985).

2) H. Kumamoto, Y. Matsubara, Y. Iizuka, K. Okamoto, and K. Yokoi, Agric. Biol. Chem., 49, 2613 (1985).

3) "The Flavonoids", ed. by J.B. Harborne, T.J. Mabry, and H. Mabry, Chapman and Hall, London (1975) p. 57.

4) G.P. Sastry, and L.R. Row, Tetrahedron, 15, 111 (1961).

5) H. Kumamoto, Y. Matsubara, Y. Iizuka, K. Okamoto, and K. Yokoi, Nippon Nogeikagaku
Kaishi, 59, 677 (1985).

6) G. Aurnhammer, H. Wangner, L. Horhamo mer, and L. Farkas, Chem. Ber., 103, 1578 (1970).

7) S. Hakomori, J. Biochem. (Tokyo), 55, 205 (1964).

温州みかん果皮中のフラボノイド配糖体 の構造及び血圧降下作用（その 2)

隈元浩康*・松原義治*。飯塚義富**。 岡本耕造***。横井勝美****

* 近畿大学理工学部応用化学科 (東大阪市小若江 3-4-1)

*** 近畿大学食品科学研究所

*** 近畿大学医学部第 1 病理学教室

**** (株) 日本サンガリア・ベバレッジ・カンパニー

著者らは前報に引き続き温州みかん果皮中のフラボノ イド配糖体について再検討し, 新たに 3 種のフラボノイ ド配糖体を単離した。各成分の構造は各種スペクトルの 測定結果から 3-ヒドロキシー $5,6,7,8,3^{\prime}, 4^{\prime}$ ーヘキサメト キシフラボン $3-\beta-\mathrm{D}-$ グルコシド (1), リモシトリン $3-$

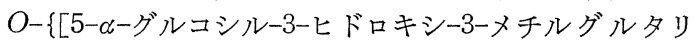

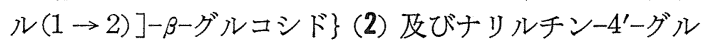
コシド (3) と決定した。また 1 及び 3 の血圧降下作用に ついて検討したところ， 1 及び 3 亿強い血圧降下作用が 認められた。本研究で単離した成分のうち 1 は新規化合 物であり， 2 及び 3 は温州みかん果皮中から初めて見い だした成分である。 